\title{
METODOLOGÍA PARA LA ESTIMACIÓN DE COSTOS DIRECTOS DE LA ATENCION INTEGRAL PARA ENFERMEDADES NO TRASMISIBLES
}

\author{
Nancy Castillo ${ }^{1, a}$, Miguel Malo 2,a, Nilda Villacres ${ }^{a}$, José Chauca $^{3, b}$, Víctor Cornetero,a, \\ Karin Roedel de Flores ${ }^{c}$, Rafaela Tapia ${ }^{d}$, Raúl Ríos ${ }^{e}$
}

\begin{abstract}
RESUMEN
Enfermedades como la diabetes mellitus (DM) y la hipertensión arterial (HTA) generan altos costos y son la causa más frecuente de mortalidad en la región de las Américas. En el caso del Perú, dados los cambios demográficos y epidemiológicos, y particularmente el aumento alarmante del sobrepeso y la obesidad, la carga de estas enfermedades tiene un crecimiento constante que implica la necesidad de asignar cada vez más recursos financieros a los servicios de salud. Los costos de la atención integral de estas enfermedades y sus complicaciones representan una carga económica que debe ser considerada por las instituciones de salud al elaborar su presupuesto. Con ese propósito, la Organización Panamericana de la Salud (OPS) ha apoyado al Ministerio de Salud (MINSA) con la realización de un estudio para la estimación de estos costos. En el presente artículo se describe esquemáticamente la metodología desarrollada para la estimación de los costos directos en la atención integral de la DM e HTA en los servicios de salud del MINSA y gobiernos regionales.
\end{abstract}

Palabras clave: Enfermedad crónica; Costos y análisis de costo; Atención integral de salud; Perú (Fuente: DeCS BIREME).

\section{METHODOLOGY FOR ESTIMATING TOTAL DIRECT COSTS OF COMPREHENSIVE CARE FOR NON-COMMUNICABLE DISEASES}

\begin{abstract}
RESUMEN
Diseases like diabetes mellitus (DM) and hypertension (HT) generate high costs and are the most common cause of mortality in the Americas. In the case of Peru, given demographic and epidemiological changes, particularly the alarming increase in overweight and obesity, the burden of these diseases is constantly increasing, resulting in the need to budget more financial resources to the health services. The total care costs of these diseases and their complications represent a financial burden that should be considered very carefully by health institutions when they draft their budgets. With this aim, the Pan American Health Organization has assisted the Ministry of Health (MINSA) with a study to estimate these costs. This article graphically describes the methodology developed to estimate the direct costs of comprehensive care for DM and HT to the health services of MINSA and regional governments
\end{abstract}

Key words: Chronic disease; Costs and cost analysis, Comprehensive Health Care; Peru (Source: MeSH NLM).

\section{INTRODUCCIÓN}

Enel Perú, lacreciente demanda paraatención del tratamiento y las complicaciones de las enfermedades no transmisibles (ENT) ${ }^{(1,2)}$, requiere de una cuidadosa estimación de costos. Para ello, la Organización Panamericana de la Salud (OPS) ha apoyado en la realización de un estudio para estimación de costos por diabetes mellitus (DM) e hipertensión arterial (HTA) en Perú. Este estudio implicó el desarrollo de una metodología que puede resultar de mucha utilidad para estimación de costos para distintos sistemas de salud de la región de las Américas.

\footnotetext{
Asociación Peruana de Obesidad y Ateroesclerosis. Lima, Perú.

Organización Panamericana de la Salud. Lima, Perú.

Ministerio de Salud. Lima, Perú.

a Doctor en Salud Pública, ${ }^{\mathrm{b}}$ magíster en Políticas Públicas, ${ }^{\mathrm{c}}$ doctor en Medicina, ${ }^{\mathrm{d}}$ bachiller en Estadística, ${ }^{\mathrm{e}}$ ingeniero informático Recibido: 17/02/2017 Aprobado: 08/03/2017 En línea: 23/03/2017
}

Citar como: Castillo N, Malo M, Villacres N, Chauca J, Cornetero V, Roedel de Flores K, Tapia R, Ríos R. Metodología para la estimación de costos directos de la atención integral para enfermedades no trasmisibles. Rev Peru Med Exp Salud Publica. 2017;34(1):119-25. doi: 10.17843/rpmesp.2017.341.2774 
En este contexto el propósito de este artículo es describir los aspectos metodológicos de la estimación de los costos directos y los requerimientos financieros para la atención integral de cuatro problemas priorizados por el Programa Presupuestal 0018 ENT, que se define como una unidad de programación de las acciones de la entidad pública, las que integradas y articuladas se orientan a proveer productos para lograr un resultado específico en la población y así contribuir al logro de un resultado final asociado a un objetivo de política pública relacionado a las ENT para el Perú.

La estimación de los costos de las ENT se establece tomando en cuenta tres aspectos: a) Los costos directos, son los gastos en atención médica desde la perspectiva de las instituciones de salud. b) Los costos indirectos, son los gastos expresados en pérdida de ingresos, productividad por muerte prematura y discapacidades en la perspectiva de los hogares, de la seguridad social y de los empleadores c) Los costos intangibles, son el resultado de costos que no fueron previstos y se calcula en cuanto se advierte un patrón de pérdida.

El presente artículo se enfoca en los costos directos que demanda la atención sanitaria que involucran todos los recursos y servicios imputados directamente a una enfermedad ${ }^{(3,4)}$.

\section{PROCESO METODOLÓGICO}

Se diseñó un proceso metodológico que consta de tres momentos:

\section{PRIMER MOMENTO: DEFINICIÓN DE GRUPOS DIAGNÓSTICOS Y PREVALENCIAS DE LAS ENT PRIORIZADAS}

Componente 1: Definición de los grupos de diagnósticos. Las morbilidades incluidas para el costeo fueron las priorizadas por el Programa Presupuestal 0018, que identifica a la DM, sobrepeso y obesidad, HTA y dislipidemia, cuyas definiciones operacionales se hicieron según los documentos normativos vigentes nacionales e internacionales ${ }^{(5-10)}$.

Componente 2: Prevalencias y cobertura. La definición de las prevalencias de los grupos definidos en el componente 1, incluyendo la población a tamizar, se construyeron a partir de estimaciones de fuentes secundarias y de estudios analíticos transversales nacionales e internacionales ${ }^{(8,11-49)}$ dado que las fuentes primarias nacionales se hallaban en etapa de corrección de subregistro ${ }^{(48)}$. La cobertura a costear se definió de acuerdo al porcentaje estimado de población asignada al subsector público de Ministerio de Salud (MINSA) $(60 \%)$. En los casos de tamizaje y tratamiento primario, la cobertura se asignó por quintil 1 y 2 de pobreza, según las definiciones operacionales del Programa Presupuestal 0018 , en otras condiciones se estimó a través de juicio de expertos.

El resultado de este ejercicio permitió estimar: 1) la población a tamizar por cada grupo etario, 2) la prevalencia para obesidad, dislipidemia, DM e HTA, 3) la prevalencia para complicaciones por DM e HTA.

El primer momento nos permite tener la lista de enfermedades, además de la prevalencia estimada para cada una de ellas y la población sana estimada sobre la cual se interviene para realizar promoción y prevención.

\section{SEGUNDO MOMENTO: ELABORACIÓN DEL MICROCOSTEO}

Componente 1: Elaboración de las condiciones / prestaciones según la historia natural de la enfermedad. En este momento metodológico se definieron las condiciones, que constituyen paquetes de prestaciones para las enfermedades definidas previamente. La definición de las condiciones de salud colectiva (promoción de la salud y prevención colectiva); y salud individual (tamizaje, tratamiento primario, y complicaciones) se muestra en la Figura 1.

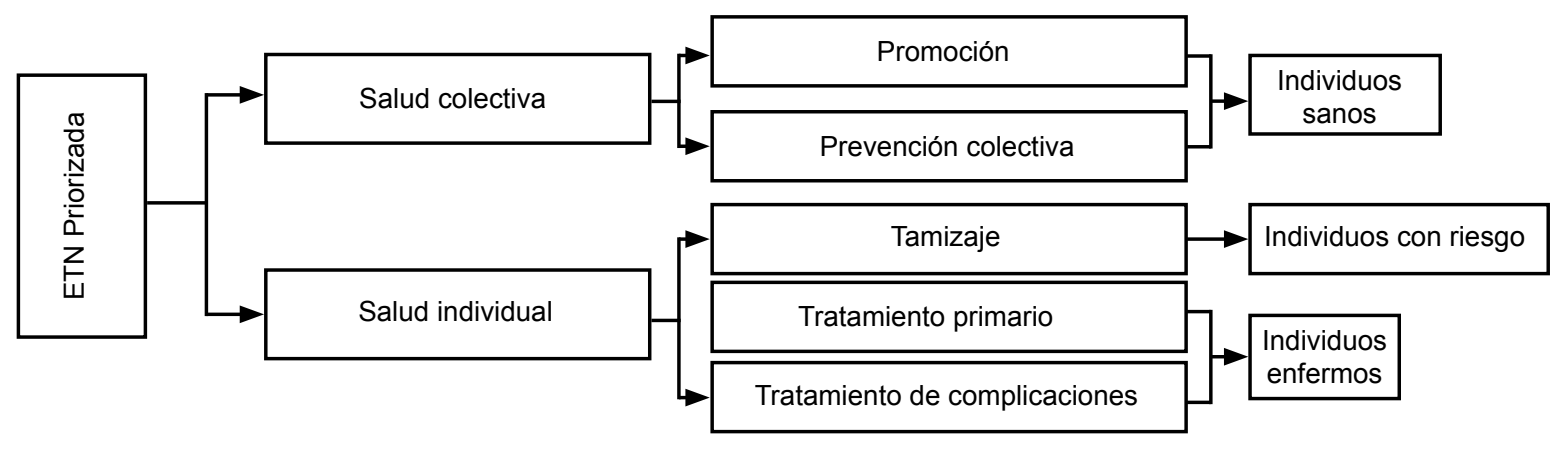

ETN: Enfermedades no transmisibles

Figura 1. Marco para la elaboración de las condiciones salud colectiva y salud individual 
Para establecer el paquete de prestaciones de cada condición se usaron las guías y protocolos aprobados por el MINSA y en caso de no existir, se utilizaron referencias internacionales que el equipo considero pertinentes y que luego valido por expertos. Esta validación de las condiciones se realizo por criterio de seis jueces expertos en el tema quienes analizaron cada ítem en función de la definición operacional dada inicialmente, estableciéndose luego el grado de acuerdo entre los jueces $(p=0.093)$.

Luego se procedió al análisis de validez de ítems, con la finalidad de precisar si cada ítem mide lo que dice medir. Para esto se usó la técnica de análisis de correlación de Pearson (para respuestas politómicas), considerándose un ítem potente y discriminante aquel con $0,5<r<1$. La confiabilidad, cuyo objetivo fue determinar la consistencia y estabilidad de las medidas en el tiempo, se precedió a determinar a través de la prueba de homogeneidad, mediante la prueba de alfa de Crombach. El baremo que se usó para estandarizar los resultados fue el modelo de escala percentilar $(œ=0,74$; confiabilidad moderadamente fuerte).

Para las condiciones de salud colectiva, en base a las directivas del MINSA, el equipo estableció 4 grupos prestaciones: 1) Promoción de salud, 2) Comunicación, 3) Gobernanza y 4) Prevención colectiva.

Para las condiciones de salud individual se siguieron los criterios de las figuras 2 y 3 .

El paquete de prestaciones para cada una de estas condiciones, se elaboró en base a las guías nacionales e internacionales y con la validación de expertos. El resultado de este ejercicio es una lista exhaustiva de prestaciones tanto de salud colectiva como de salud individual que se constituye en la base de información para la estimación de costos y se agrupan en siete categorías:

1. Salud colectiva: que incluye a la DM e HTA.

2. Prevención: población sana, objetivo para el tamizaje de factores de riesgo para DM, HTA, dislipidemia y obesidad.

3. Tratamiento de la DM.

4. Complicaciones de la DM: macrovasculares y microvasculares.

5. Tratamiento de la HTA.

6. Complicaciones de la HTA: macrovasculares y microvasculares.

7. Obesidad / dislipidemia: población diagnosticada de obesidad o dislipidemia en el tamizaje.

Componente 2: Elaboración de estructuras de costos. Los costos unitarios, que incluyen los costos fijos y variables, se establecieron después de la definición de las prestaciones descrita en el componente 1, considerando para cada prestación el primer, segundo y tercer nivel de atención de salud. En el caso del Perú, para estimar los costos de los materiales e insumos se utilizaron los precios del tarifario del Seguro Integral de Salud (SIS) con Resolución Jefatural 082-2014/SIS y $139-2014 /$ SIS $^{(50,51)}$ y como referencia en la actualización se revisó la Resolución Jefatural $011-2016^{(52)}$ y se contó con la información de la cartera de precios que presenta el programa presupuestal $0018{ }^{(53)}$. Para los precios de los medicamentos se recurrió al portal del Observatorio Peruano de Medicamentos ${ }^{(49)}$. El costo de los recursos humanos se calculó a partir del salario integrado neto anualizado, tras considerar tanto las prestaciones sociales como otros beneficios, como aguinaldos y bonos de acuerdo a los decretos legislativos recibidos por el

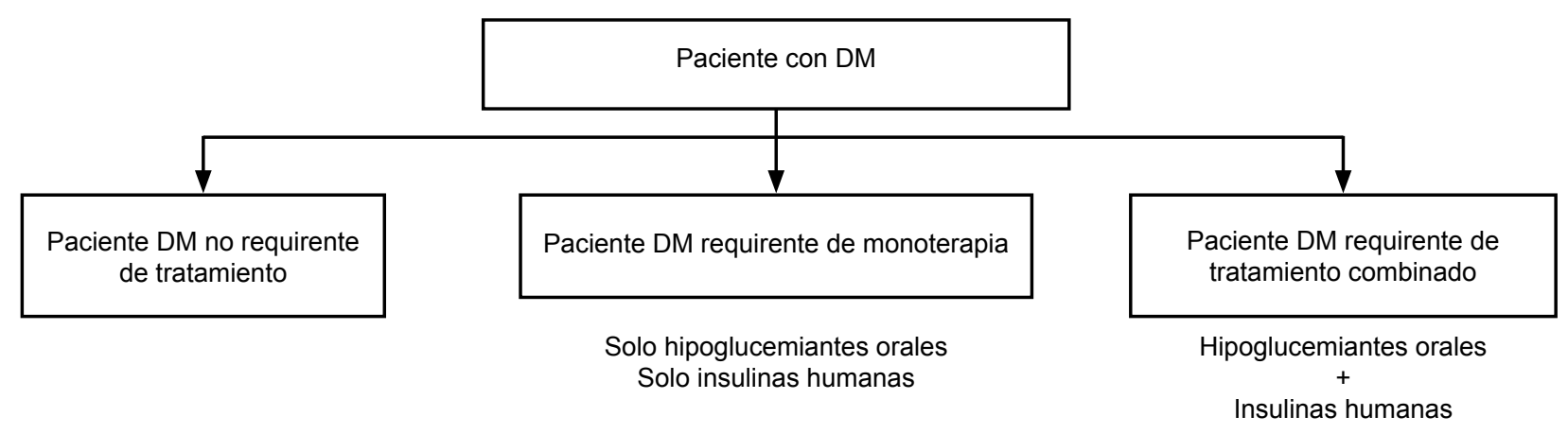

DM: diabetes mellitus

Valoración Inicial: Evaluación única en primera consulta del año para valoración de otros factores de riesgo Seguimiento: Evaluación continua con medición de al menos cuatro controles anuales

Tratamiento: indicación farmacológica según el grupo correspondiente a valorizar

Figura 2. Salud Individual. Tratamiento primario de la diabetes mellitus. 


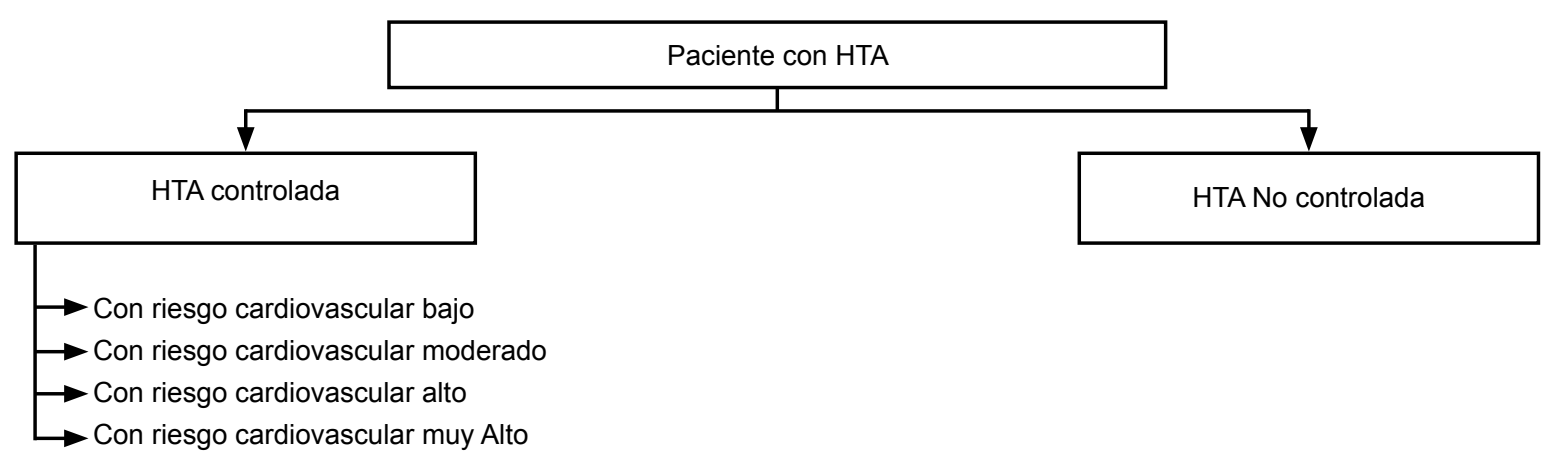

HTA: hipertensión arterial

Valoración Inicial: Evaluación única en primera consulta del año para valoración de otros factores de riesgo

Seguimiento: Evaluación continua con medición de al menos cuatro controles anuales

Tratamiento: indicación farmacológica según el grupo correspondiente a valorizar

Figura 3. Salud Individual. Tratamiento primario de la Hipertensión Arterial

trabajador, los mismos que fueron convertidos en hora/ costo y minuto/costo. Los costos de los activos fijos (inmuebles, equipo e instrumental médico) se depreciaron y prorratearon de acuerdo con la metodología de costos estándar del MINSA RM 197- $2009{ }^{(53) .}$

La atención médica se estructuró para cinco eventos relevantes: a) consulta externa- ambulatoria; b) de emergencias; c) hospitalaria; d) intervención quirúrgica y e) en unidad de cuidados intensivos o unidad especializada.

De manera que al momento contamos con cuatro elementos: enfermedad, población a intervenir, prestaciones y costo para cada prestación.

Componente 3: Estimación del per cápita. Para la estimación de la unidad per cápita se estiman los costos por módulos de condiciones y población objetivo según prevalencia y cobertura para un período de un año. Se divide el cálculo por prestaciones colectivas: (promoción de la salud y prevención colectiva) y prestaciones individuales (tratamiento primario y de las complicaciones) y se establece los costos por cada condición y prestación.

Para las condiciones colectivas se tomaron las definiciones operacionales del programa presupuestal ENT $0018{ }^{(52)}$ y sus criterios de programación de población. Dependiendo del tipo de intervención (sesiones, consejería, visitas, talleres, etc.) se determina la población objetivo (familias, instituciones educativas, distritos, comunidades, etc.)

Para el cálculo del per cápita de las condiciones de salud individual se calcula primero el costo de cada condición de salud, sumando el costo de cada paquete de procedimientos médicos y esquemas de tratamiento (descritos en cada condición de acuerdo a la normativa vigente nacional e internacional) multiplicado por el número de atenciones anuales por paciente.

\section{Costo de condición de salud = Sumatoria de procedimientos médicos $x$ frecuencia anual de entrega procedimientos}

Una vez determinado el costo de la condición de salud, este se multiplica por la cantidad de población que corresponde a la estimación de prevalencia para cada condición.

Costo de condición $\times$ Prevalencia de la condición = valorización (costo) total de las condiciones de salud

Finalmente, para calcular el costo percápita unitario por DM e HTA, se divide el costo de cada condición para la población afectada, que es la prevalencia de la población con diabetes mellitus e hipertensión arterial.

Sumatoria de valorización (costo) de las condiciones de salud / número de población objetivo que recibe la intervención = costo unitario per cápita especifico

\section{TERCER MOMENTO: FINANCIAMIENTO}

El costo directo total de las intervenciones de DM e HTA se estiman en alrededor de 4688 millones de soles, lo que equivale al $18,1 \%$ del total de presupuesto del sub sector público, gobiernos regionales y el MINSA. El per cápita por el total de la población del país es de S/ 149; pero si calculamos el per cápita por la población afectada este alcanza más de S/ 2860 por persona.

Los recursos financieros invertidos (Perú, 2016) en DM e HTA son aproximadamente S/ 1557,5 millones 
Tabla 1. Proceso metodológico para la estimación de costos en enfermedades no transmisibles priorizadas

\begin{tabular}{ll}
\hline Momentos & \multicolumn{1}{c}{ Componentes } \\
\hline $\begin{array}{l}\text { Primer momento: Definición de grupos diagnósticos y } \\
\text { prevalencias de las ENT priorizadas }\end{array}$ & $\begin{array}{l}\text { Componente 1: Definición de los grupos de diagnósticos y } \\
\text { prestaciones identificados en el estudio } \\
\text { Componente 2: Prevalencias y cobertura. }\end{array}$ \\
& $\begin{array}{l}\text { Componente 1: Elaboración de las condiciones / prestaciones según } \\
\text { la historia natural de la enfermedad } \\
\text { Componente 2: Elaboración de estructuras de costos } \\
\text { Cegundo momento: Análisis del microcosteo }\end{array}$ \\
Componente 3: Estimación del per cápita \\
Tercer momento: Financiamiento por escenarios. & $\begin{array}{l}\text { Componente 1: Financiamiento } \\
\text { Componente 2: Escenarios epidemiológicos/económicos }\end{array}$ \\
\hline
\end{tabular}

ENT: Enfermedades no transmisibles

de soles lo que equivale al $6 \%$ del total de presupuesto de Salud; de acuerdo a las estimaciones de costos en DM e HTA el presupuesto requerido alcanzaría el $18 \%$ del total del presupuesto de salud del subsector público, gobiernos regionales y el MINSA, lo que nos deja una brecha de casi $12 \%$ por cerrar.

La salud colectiva más el tamizaje representan el $15 \%$ de la valorización total de las intervenciones en DM e HTA, y el $85 \%$ corresponde al diagnóstico, tratamiento y a las complicaciones de estas enfermedades.

Una vez establecidos los costos directos de la atención integral de la DM y la HTA se procede a la estimación de los requerimientos financieros para el abordaje integral de la enfermedad; es decir, en el caso peruano para financiar los productos del programa presupuestal relacionados con la DM y la HTA. El equipo decidió hacer la proyección de esta estimación hasta el año 2030.

Para estimar este requerimiento se tomaron en cuenta cuatro variables: el crecimiento económico, el gasto en salud, el crecimiento poblacional y las tendencias de prevalencia que dependerán de las tres anteriores variables.

En el caso peruano se consideró un crecimiento del producto bruto interno (PIB) que va desde $3,2 \%$ entre el 2016 y el 2018 a un $6,1 \%$ entre el 2026 y el 2030 (55-57). Así mismo, se consideró el supuesto del incremento del gasto en salud de un $4,9 \%$ en el 2015 a un $7,5 \%$ del PBI al año 2030 (55-57).

En relación al crecimiento poblacional se tomaron los datos al año 2030 del cuadro población estimado y proyectado por sexo y tasa de crecimiento, según años calendarios, 2000
- 2050; del Instituto Nacional de Estadística e Informática (INEI) ${ }^{(58)}$. En este caso se optó por un escenario favorable que implica la disposición de un mayor presupuesto para salud y por tanto, el comportamiento de los indicadores de DM e HTA se estimaría a la baja. Esta definición de escenarios debería tomar en cuenta las condiciones políticas y económicas de cada país.

Es importante que se definan políticas de salud en las que se priorice las intervenciones más costo efectivas como son las de salud colectiva y tamizaje, así como el presupuesto de una atención primaria que permita reducir la incidencia de ambas enfermedades.

\section{CONCLUSIONES}

Tomando en cuenta los resultados obtenidos en la estimación de costos a partir de la metodología descrita, consideramos que este trabajo realizado en el Perú, es un valioso instrumento para la gestión sanitaria en la región, pues implica el desarrollo de una metodología que tiene un doble valor: por un lado, permite superar las deficiencias con los sistemas de información en salud, así como las limitaciones en relación a la normativa nacional de salud que todavía existen en países de la región de las Américas ${ }^{(59)}$ articulando distintas fuentes de información primarias y secundarias a nivel nacional e internacional, haciendo posible una estimación de costos más cercana a las condiciones particulares de cada contexto. Y, por otro lado, la posibilidad de que los conocimientos en economía de la salud, entre ellos la estimación de costos por ENT estén cada vez más accesibles a profesionales de la Salud Pública, y que puedan ser utilizados en los distintos niveles del sistema de salud, fortaleciendo su respuesta al desafío creciente de las ENT.

\section{REFERENCIAS BIBLIOGRÁFICAS}

1. Lim SS, Vos T, Flaxman AD, Danaei G, Shibuya K, Adair-Rohani $\mathrm{H}$, et al. A comparative risk assessment of burden of disease and injury attributable to 67 risk factors and risk factor clusters in 21 regions, 19902010: a systematic analysis for the Global Burden of Disease Study 2010.
Lancet. 2012;380(9859):2224-60. doi: 10.1016/S0140-6736(12)61766-8.

2. Organización Panamericana de la Salud (OPS). Perú: Estudio Estimación de 
Costes Directos de la Atención Integral de la DM2 e HTA. Lima: OPS; 2015.

3. Choi B, Pak A, Ottoson J. Understanding the basic concepts of public health surveillance. J Epidemiol Community Health. 2002;56(6):402. doi: 10.1136/ jech.56.6.402

4. Evers SMAA, Struijs JN, Ament AJHA, Genugten MLL van, Jager JC, Bos GAM van den. International comparison of stroke cost studies. Stroke. 2004;35:1209-15. doi: 10.1161/01. STR.0000125860.48180.48

5. American Diabetes Association. 12. Management of diabetes in pregnancy. Diabetes care. 2016;39 Suppl 1:S94-8. doi: $10.2337 / \mathrm{dc} 16-S 015$

6. Egan AM, Murphy HR, Dunne FP. The management of type 1 and type 2 diabetes in pregnancy. QJM. 2015;108(12):923 7. doi: $10.1093 /$ qjimed/hcv060

7. Ministerio de Salud del Perú. Guía Técnica: Guía de práctica clínica para el diagnóstico, tratamiento y control de la diabetes mellitus tipo 2 en el primer nivel de atención. Lima: MINSA; 2016.

8. Ministerio de Salud del Perú. Guía Técnica: Guía práctica clínica para el diagnóstico, tratamiento y control de la enfermedad hipertensiva. Lima: MINSA; 2015.

9. Comments on the $2013 \mathrm{ESC} / \mathrm{ESH}$ guidelines for the management of arterial hypertension 2013. A report of the Task Force of the Clinical Practice Guidelines Committee of the Spanish Society of Cardiology. Re Esp Cardiol. 2013;66(11):842-7.

10. Hallan SI, Dahl K, Oien CM, Grootendorst DC, Aasberg A, Holmen $\mathrm{J}$, et al. Screening strategies for chronic kidney disease in the general population: follow-up of cross sectional health survey. BMJ. 2006;333(7577):1047. doi: 10.1136/bmj.39001.657755.BE

11. Álvarez-Dongo D, Sánchez-Abanto J, Gómez-Guizado G, Tarqui-Mamani C. Sobrepeso y obesidad: prevalencia y determinantes sociales del exceso de peso en la población peruana (20092010). Rev Peru Med Exp Salud Publica. 2012;29(3):303-13.

12. Instituto Nacional de Estadística e Informática. Encuesta Nacional de Demografía y de Salud - ENDES [Internet]. Lima: INEI; 2015 [citado el 25 de noviembre de 2016]. Disponible en: http://iinei.inei.gob.pe/microdatos

13. Carrillo-Larco RM, Bernabe-Ortiz A, Pillay TD, Gilman RH, Sanchez JF,
Poterico JA, et al. Obesity risk in rural, urban and rural-to-urban migrants: prospective results of the PERU MIGRANT study. Int J Obes (Lond). 2016;40(1):181-5.

14. Benziger CP, Bernabé-Ortiz A, Gilman RH, Ceckely W, Smeeth L, Malaga G, et al. Metabolic Abnormalities Are Common among South American Hispanics Subjects wiht Normal Weight or Excercise or Excess Body Weigth: The CRONICAS Cohort Study. PloS one. 2015;10(11):e0138968.

15. Poterico JA, Bernabe-Ortiz A, Loret de Mola C, Miranda JJ. [Association between television viewing and obesity in Peruvian women]. Rev Saude Publica. 2012;46(4):610-6.

16. Lazo-Porras M, Bernabe-Ortiz A, Malaga G, Gilman RH, Acuna-Villaorduna A, Cardenas-Montero D, et al. Low HDL cholesterol as a cardiovascular risk factor in rural, urban, and rural-urban migrants: PERU MIGRANT cohort study. Atherosclerosis. 2016;246:36-43.

17. International Diabetes Federation. IDF Diabetes Atlas. 7 ed. Brussels, Belgium: International Diabetes Federation; 2015.

18. Seclen SN, Rosas ME, Arias AJ, Huayta E, Medina CA. Prevalence of diabetes and impaired fasting glucose in Peru: report from PERUDIAB, a national urban population-based longitudinal study. BMJ Open Diabetes Res Care. 2015;3(1):e000110.

19. Instituto Nacional de Estadística e Informática. Perú: Enfermedades No Transmisibles y Transmisibles, 2014. Lima: INEI; 2014.

20. Dirección de Prevención de Enfermedades No Transmisibles y Oncológicas, Ministerio de salud del Perú. Guía de Práctica Clínica para el Diagnóstico, Tratamiento y Control de la Diabetes Mellitus Tipo 2 en el Primer Nivel de Atención. Lima: Ministerio de Salud; 2016

21. Segura Vega L, Agustí R, Ruiz Mori E. La hipertensión arterial en el Perú según el estudio TORNASOL II. Rev Peru Cardiol. 2011;37(1):19-27.

22. Román-Vargas JA, Vázquez-Martínez $\mathrm{VH}$, Loera-Morales J, Cantú-Solís ON, Cervantes-Vázquez DA, BernabéAdame CC. Epidemiological outlook of the patient with uncontrolled systemic hypertension in a family medicine Unit in Reynosa, Tamaulipas panorama epidemiológico do paciente com hipertensão não controlada em uma unidade de medicina de família de Reynosa, Tamaulipas. Aten Fam. 2016;23(1):14-8.

23. American Diabetes Association Standards of Medical Care in Diabetes-2016 Abridged for Primary Care Providers. Clin Diabetes. 2016;34(1):3-21.

24. Garber AJ, Abrahamson MJ, Barzilay JI, Blonde L, Bloomgarden ZT, Bush MA, et al. Consensus Statement by the American Association of Clinical Endocrinologists and American College of Endocrinology on the Comprehensive Type 2 Diabetes Management Algorithm--2016 Executive Summary. Endocr Pract. 2016;22(1):84-113.

25. Haukka J, Hoti F, Erasto P, Saukkonen T, Makimattila S, Korhonen P. Evaluation of the incidence and risk of hypoglycemic coma associated with selection of basal insulin in the treatment of diabetes: a Finnish register linkage study. Pharmacoepidemiol Drug Saf. 2013;22(12):1326-35.

26. Solanki JD, Makwana AH, Mehta HB, Gokhale PA, Shah CJ. A Study of prevalence and association of risk factors for diabetic vasculopathy in an urban area of Gujarat. J Family Med Prim Care. 2013;2(4):360-4.

27. Castañeda-Guarderas A, Beltrán-Ale G, Casma-Bustamante R, Ruiz-Grosso P, Málaga G. Registro de pacientes con accidente cerebro vascular en un hospital público del Perú, 2000-2009. Rev Peru Med Exp Salud Publica. 201 1;28(4):62327.

28. Balan D, Babes PA. Incidence and type of stroke in patients with diabetes. Comparison between diabetics and nondiabetics. Rom J Intern Med. 2009;47(3):249-55.

29. Hoffman DL, Sadosky A, Alvir J. Crossnational burden of painful diabetic peripheral neuropathy in Asia, Latin America, and the Middle East. Pain Pract. 2009;9(1):35-42.

30. Margolis DJ, Malay DS, Hoffstad OJ, Leonard CE, MaCurdy T, Lopez de Nava K, et al. Prevalence of diabetes, diabetic foot ulcer, and lower extremity amputation among Medicare beneficiaries, 2006 to 2008: Data Points \#1. In: Data Points Publication Series [Internet]. Rockville (MD): Agency for Healthcare Research and Quality (US); 2011 [citado el 23 de noviembre de 2016]. Disponible en: https://www.ncbi. nlm.nih.gov/books/NBK63602/ 
31. Fei YF, Wang C, Chen DW, Li YH, Lin S, Liu GJ, et al. [Incidence and risk factors of amputation among in patients with diabetic foot]. Zhonghua Yi Xue Za Zhi. 2012;92(24):1686-9. [Article in Chinese]

32. Krasnik V, Stefanickova J, Fabkova J, Buckova D, Helbich M. [Prevalence of the Diabetic Retinopathy and Genetic Factors Significance in the Development of Diabetic Retinopathy in Patients with Diabetes Mellitus type I and II in Slovakia (DIARET SK study). Overview of Actual Findings and Design of the Epidemiological DIARET SK Study]. Cesk Slov Oftalmol. 2015;71(5):237-42. [Article in Czech]

33. Lee R, Wong TY, Sabanayagam C. Epidemiology of diabetic retinopathy, diabetic macular edema and related vision loss. Eye Vis (Lond). 2015;2:17.

34. Herrera-Añazco P, Hernández AV, Mezones-Holguin E. Diabetes Mellitus y Nefropatia Diabética en el Perú. Nefrología, Diálisis y Trasplante. 2015;35(4):229-37.

35. Alcazar Arroyo R, Orte Martínez L, Otero González A. enfermedad renal crónica avanzada. Nefrología (Madr). 2008;28 Supl. 3:3-6.

36. Tanahashi N. [Hypertension associated with cerebrovascular disease]. Nihon Rinsho. 2015;73(11):1864-70. Article in Japanese]

37. Herrera-Añazco P, Benites-Zapata VA, León-Yurivilca I, Huarcaya-Cotaquispe R, Silveira-Chau M. Chronic kidney disease in Peru: a challenge for a country with an emerging economy.J Bras Nefrol. 2015;37(4):507-8.

38. McCullough K, Sharma P, Ali T, Khan I, Smith WC, MacLeod A, et al. Measuring the population burden of chronic kidney disease: a systematic literature review of the estimated prevalence of impaired kidney function. Nephrol Dial Transplant. 2012;27(5):1812-21.

39. Cusumano AM, Rosa-Diez GJ, Gonzalez-Bedat MC. Latin American Dialysis and Transplant Registry: Experience and contributions to endstage renal disease epidemiology. World J Nephrol. 2016;5(5):389-97.

40. Bell EK, Gao L, Judd S, Glasser SP, McClellan W, Gutierrez OM, et al. Blood pressure indexes and end-stage renal disease risk in adults with chronic kidney disease. Am J Hypertens. 2012;25(7):789-96.
41. Carlini R, Obrador G, Campistrús N, Andrade L, Chifflet L, Bregman $\mathrm{R}$, et al. The First Report of The Latin American Society of Nephrology and Hypertension (SLANH) Anemia Committee in Chronic Hemodialysis Patients. Nefrologia. 2014;34(1):96-104.

42. Dirección Nacional de Normatización, Ministerio de Salud Pública del Ecuador. Diagnóstico y tratamiento de la diabetes en el embarazo (pre-gestacional y gestacional). Guía de Práctica Clínica. Quito: Ministerio de Salud Pública del Ecuador; 2014.

43. Callec R, Perdriolle-Galet E, Sery GA, Lamy C, Floriot M, Fresson J, et al. [Type 2 diabetes and pregnancy: epidemiology and obstetrical consequences. A 97 women continuous series]. J Gynecol Obstet Biol Reproduction (Paris). 2015;44(1):41-6.

44. Feldman AZ, Brown FM. Management of Type 1 Diabetes in Pregnancy. Curr Diab Rep. 2016;16(8):76.

45. Lapolla A, Dalfra M. Pregnancy complicated by type 2 diabetes: an emerging problem. Diabetes Res Clin Pract. 2008;80(1):2-7.

46. Zisser HC, Biersmith MA, Jovanovic LB, Yogev Y, Hod M, Kovatchev BP. Fetal risk assessment in pregnancies complicated by diabetes mellitus. J Diabetes Sci Technol. 2010;4(6):1368-73.

47. Evers IM, de Valk HW, Visser GH. Risk of complications of pregnancy in women with type 1 diabetes: nationwide prospective study in the Netherlands. BMJ. 2004;328(7445):915.

48. Ministerio de Salud del Perú. Indicadores de Monitoreo de la Información HIS 3.05 [Internet]. Lima: Ministerio de Salud; 2013 [citado el 25 de noviembre de 2016]. Disponible en: http://bvs. minsa.gob.pe/local/MINSA/2737.pdf

49. Dirección General de Medicamentos Insumos y Drogas, Ministerio de Salud del Perú. Observatorio de productos farmacéuticos: Módulo de consulta de precios [Internet]. Lima: Ministerio de Salud; c2016 [citado el 25 de noviembre de 2016]. Disponible en: http:// observatorio.digemid.minsa.gob.pe/

50. Seguro Integral de Salud, Ministerio de Salud del Perú. Resolución Jefatural ${ }^{\circ}$ 082-2014/SIS. Lima, Perú 27 de marzo de 2014 .

51. Seguro Integral de Salud, Ministerio de Salud del Perú. Resolución Jefatural $\mathrm{N}^{\circ}$ 139-2014/SIS. Lima, Perú 23 de junio de 2014.
52. Seguro Integral de Salud, Ministerio de Salud del Perú. Resolución Jefatural $\mathrm{N}^{\circ}$ 011-2016/SIS. Lima, Perú 13 de enero de 2016.

53. Ministerio de Salud del Perú. Programa Presupuestal 0018: Enfermedades No Transmisibles. Lima: MINSA; 2016.

54. Ministerio de Salud del Perú. Resolución Ministerial $\mathrm{N}^{\mathrm{a}} \quad$ 195-2009/MINSA. Metodología para la estimación de costos estándar en los establecimientos de salud. Lima, Perú 27 de marzo de 2009.

55. Agreda V, Alegría R, Artaza MI, Boix JV, Casanueva H, Cordeiro JL, et al. Latinoamérica 2030: Estudio Delphi y Escenarios[Internet]. The Millennium Project; 2012 [citado el 25 de noviembre de 2016]. Disponible en: http://www. prospectivayestrategia.cl/pdf/2030.pdf

56. Bonifaz JL, Casas C, Sanborn CA, Seminario B, Urrunaga R, Vásquez E, et al. El Perú hacia el 2062: pensando juntos en el futuro. Lima. Universidad del Pacifico; 2013.

57. Ministerio del Ambiente del Perú. Anexo: Supuestos y metodologías contempladas como parte de las contribuciones nacionales en mitigación [Internet]. Lima: Ministerio del Ambiente; 2015 [citado el 25 de noviembre de 2016]. Disponible en: http://www.minam. gob.pe/indcs/wp-content/uploads/ sites/100/2015/06/Supuestos-yMetodolog\%C3\%ADas-contempladascomo-parte-de-las-ContribucionesNacionales-en-Mitigaci\%C3\%B3n.pdf

58. Dirección Técnica de Demografía e Indicadores Sociales, Instituto Nacional de Estadística e Informática. Perú: Estimaciones y Proyecciones de Población 1950-2050. Boletín de Análisis Demográfico $\mathrm{N}^{\circ}$ 36. Lima: INEI; 2009

59. Organización Panamericana de la Salud. Situación de Salud en las Américas: Indicadores Básicos 2011 [Internet]. Washington, DC: OPS; 2011 [citado el 25 de noviembre de 2016]. Disponible en: http://ais.paho.org/chi/ brochures/2011/BI_2011_ESP.pdf

Correspondencia: Nancy Castillo Martínez Dirección: Av. Jorge Chavez 468 Dpto. 3 Barranco. Lima, Perú Teléfono: (511) 993594608 Correo electrónico: pahuje@hotmail.com 